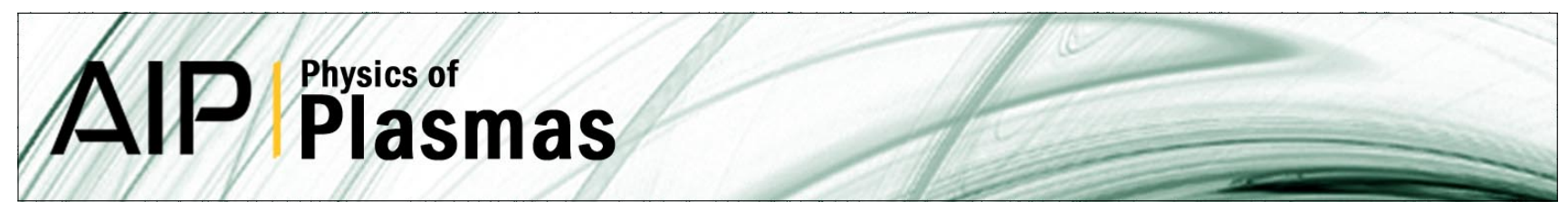

\title{
Indeterminacy and instability in Petschek reconnection
}

Terry G. Forbes, Eric R. Priest, Daniel B. Seaton, and Yuri E. Litvinenko

Citation: Phys. Plasmas 20, 052902 (2013); doi: 10.1063/1.4804337

View online: http://dx.doi.org/10.1063/1.4804337

View Table of Contents: http://pop.aip.org/resource/1/PHPAEN/v20/i5

Published by the AIP Publishing LLC.

\section{Additional information on Phys. Plasmas}

Journal Homepage: http://pop.aip.org/

Journal Information: http://pop.aip.org/about/about_the_journal

Top downloads: http://pop.aip.org/features/most_downloaded

Information for Authors: http://pop.aip.org/authors

\section{ADVERTISEMENT}

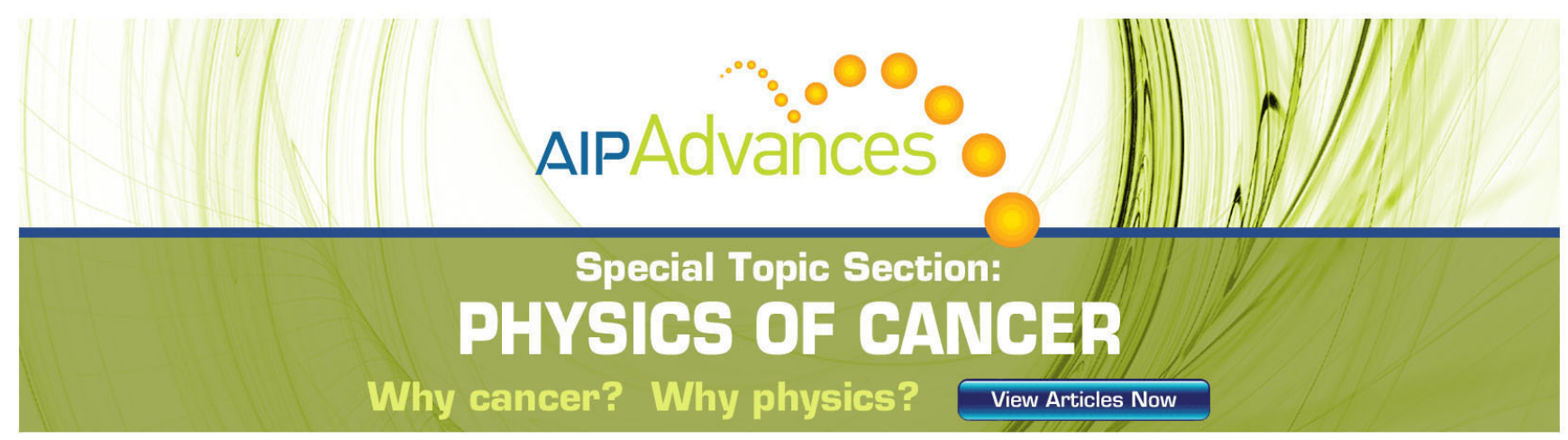




\title{
Indeterminacy and instability in Petschek reconnection
}

\author{
Terry G. Forbes, ${ }^{1}$ Eric R. Priest, ${ }^{2}$ Daniel B. Seaton, ${ }^{3}$ and Yuri E. Litvinenko ${ }^{4}$ \\ ${ }^{1}$ Institute for the Study of Earth, Oceans, and Space, University of New Hampshire, Durham, \\ New Hampshire 03824, USA \\ ${ }^{2}$ Institute of Mathematics, University of St. Andrews, Fife KY16 9SS, Scotland \\ ${ }^{3}$ SIDC-Royal Observatory of Belgium, Ringlaan 3, 1180 Brussels, Belgium \\ ${ }^{4}$ Department of Mathematics, University of Waikato, P.O. 3105, Hamilton, New Zealand
}

(Received 21 January 2013; accepted 22 April 2013; published online 13 May 2013)

\begin{abstract}
We explain two puzzling aspects of Petschek's model for fast reconnection. One is its failure to occur in plasma simulations with uniform resistivity. The other is its inability to provide anything more than an upper limit for the reconnection rate. We have found that previously published analytical solutions based on Petschek's model are structurally unstable if the electrical resistivity is uniform. The structural instability is associated with the presence of an essential singularity at the $\mathrm{X}$-line that is unphysical. By requiring that such a singularity does not exist, we obtain a formula that predicts a specific rate of reconnection. For uniform resistivity, reconnection can only occur at the slow, Sweet-Parker rate. For nonuniform resistivity, reconnection can occur at a much faster rate provided that the resistivity profile is not too flat near the X-line. If this condition is satisfied, then the scale length of the nonuniformity determines the reconnection rate. (C) 2013 AIP Publishing LLC. [http://dx.doi.org/10.1063/1.4804337]
\end{abstract}

\section{INTRODUCTION}

When Petschek's model ${ }^{1}$ was first introduced it was thought by many to provide a universal description of fast reconnection in a highly conducting plasma. With the development of computer simulations, it became apparent that Petschek's model only occurs in certain situations. For example, it occurs when the electrical resistivity in the vicinity of the X-line is locally enhanced. ${ }^{2-6}$ More recently, Baty et al. ${ }^{7,8}$ have found that Petschek reconnection can also be generated using a nonuniform viscosity profile ${ }^{8}$ or imposing an asymmetric, nonuniform resistivity in the form of a simple step function. ${ }^{7}$ The possible conditions that can produce Petschek reconnection are, therefore, more varied than is commonly realized.

Another aspect of Petschek's model that is often not appreciated is that it does not predict a specific reconnection rate but only a range of possible values. The lower value of this range is the Sweet-Parker rate of $M_{A}=R_{m}^{-1 / 2}$, where $M_{A}$ is the inflow Alfvén Mach number and $R_{m}$ is the magnetic Reynolds (or Lundquist) number. The upper value of the range is the fast rate given by Petschek's well-known formula $M_{A}=\pi /\left(8 \log R_{m}\right){ }^{1}$ The indeterminacy of the reconnection rate in Petschek's model means that some additional assumption must be made to obtain a specific value. Some authors simply assume that the rate will occur at the fastest possible value, ${ }^{9}$ while others assume that the rate is set by the inflow boundary conditions. ${ }^{10}$ The idea that the reconnection will occur at the fastest possible rate presupposes that a configuration with a slow rate is less stable than one with a fast rate. However, numerical simulations using a uniform resistivity show just the opposite behavior. Even if one starts with a fast configuration, it rapidly evolves to the slow Sweet-Parker configuration ${ }^{11}$ suggesting that it is the fast-rate configuration that is unstable. The alternate idea that the inflow boundary conditions set the rate is also problematical. Petschek's model assumes the reconnection process to be undriven. ${ }^{12}$ If the reconnection is undriven, then the inflow speed cannot be imposed, but must, instead, be calculated self consistently from the equations.

Within the last 10 years, Malyshkin ${ }^{13,14}$ and Kulsrud ${ }^{15}$ have argued that the ambiguity of the reconnection rate in Petschek's model is due to the inconsistency of the model with Faraday's equation. They claim that if this inconsistency is removed, then the reconnection rate is uniquely determined to be the slow, Sweet-Parker rate in the case of uniform resistivity. Their analysis, however, appears to contradict earlier analyses by Vasyliunas, ${ }^{16}$ Somov, ${ }^{17,18}$ and Titov,${ }^{19,20}$ which do not show any inconsistency.

The previous analyses by Vasyliunas, ${ }^{16}$ Somov, ${ }^{17,18}$ and Titov, ${ }^{19,20}$ as well as the newer ones by Malyshkin and Kulsrud, ${ }^{13-15}$ are based on the one-dimensional system of equations obtained by averaging the steady-state, twodimensional resistive MHD equations over the thickness of the reconnection current layer, including both the diffusion and slow shock regions. The one-dimensional equations that result provide a description of the average properties of the plasma along the outflow direction. In the present paper, we develop an analytical framework that allows us to compare the earlier analyses with the newer ones by Malyshykin and Kulsrud. We show that, although the older analyses are consistent with Faraday's equation, they generally imply the existence of an unphysical singularity at the X-line. Eliminating these singular solutions leads to a specific value for the reconnection rate that is roughly similar to that predicted by Malyshykin and Kulsrud.

In order to obtain some physical insight into the nature of the singularity at the $\mathrm{X}$-line, we extend the previous analyses by Vasyliunas and Somov to include time-dependence. We find that the unphysicality of the singular solutions is 
closely related to the fact that they are structurally unstable in time. (Solutions that are unstable in this way are also called nonevolutionary. ${ }^{21}$ ) If the singular solutions are smoothed, for example, by finite differencing, they no longer correspond to steady-state solutions. Such smoothed solutions will rapidly evolve to a nonsingular solution if one exists. If a nonsingular solution does not exist, then the initial configuration never reaches a steady state. The behavior of our time-dependent system is remarkably similar to that observed in the numerical simulations using the twodimensional resistive MHD equations with uniform and nonuniform resistivity.

In Sec. II, we derive time-dependent versions of the MHD equations previously obtained by Vasyliunas, ${ }^{16}$ Somov, ${ }^{18}$ and Titov. ${ }^{19}$ Then in Sec. III, we consider the steady-state solutions to these equations and compare them to those of Malyshykin and Kulsrud. In Sec. IV, we consider the stability of the steady-state solutions by numerically solving the time-dependent equations. In Sec. V, we develop a more realistic treatment of the diffusion region that avoids some of the simplifying assumptions made in the previous analyses. Finally, in Sec. VI we discuss the physical implications of our analysis.

\section{AVERAGED RESISTIVE MHD EQUATIONS}

The idea of simplifying the reconnection problem by averaging the MHD equations over the reconnection current layer was first considered by Vasyliunas. ${ }^{16}$ He obtained an equation for the average outflow velocity assuming incompressibility and an approximately uniform external magnetic field. Ten years later Somov and Titov carried out a similar averaging procedure, but they allowed for compressibility. ${ }^{17-20}$ They also included a nonuniform external field based on the model of Green $^{22}$ and Syrovatskii. ${ }^{23}$ (For a step-by-step derivation of the Somov and Titov equations see Seaton and Forbes. ${ }^{24}$ ) The equation obtained by Somov and Titov reduces to Vasyliunas's equation in the limit that the plasma $\beta$ and the length of the current layer both become infinite. Our analysis most closely follows that of Somov and Titov and uses their notation and coordinates. For simplicity, we only consider the incompressible limit, but, unlike Vasyliunas and Somov and Titov, we include time-dependence. The equations we obtain for the averaged quantities are closely related to the one-dimensional MHD nozzle equations. ${ }^{25}$ These equations provide a simplified description of the average plasma properties along the length of the flow, and they are often used to model astrophysical jets. $^{26,27}$

To obtain the nozzle equations, we start with the timedependent, two-dimensional resistive MHD equations in the incompressible limit:

$$
\begin{gathered}
B_{t}=-E_{y}, \\
b_{t}=E_{x}, \\
E=-V b+u B+\eta(x)\left(b_{x}-B_{y}\right), \\
V_{x}+u_{y}=0, \\
B_{x}+b_{y}=0,
\end{gathered}
$$

$$
\begin{gathered}
V_{t}+V V_{x}+u V_{y}=-p_{x}-\left(B^{2}+b^{2}\right)_{x} / 2+B B_{x}+b B_{y} \\
u_{t}+V u_{x}+u u_{y}=-p_{y}-\left(B^{2}+b^{2}\right)_{y} / 2+B b_{x}+b b_{y}
\end{gathered}
$$

where $B$ and $b$ are the $x$-and $y$-components of the magnetic field, $V$ and $u$ are the $x$ - and $y$-components of the bulk flow, $E$ is the magnitude of the electric field (in the z-direction), $\eta(x)$ is a spatially variable magnetic diffusivity, $p$ is the gas pressure, and the subscripts indicate partial derivates with respect to $t, x$, and $y$. These equations have been made dimensionless by normalizing $B$ and $b$ to $B\left(0, a_{0}\right), V$ and $u$ to $V_{A}=B\left(0, a_{0}\right) / \sqrt{4 \pi \rho}, p$ to $B\left(0, a_{0}\right)^{2} / 4 \pi \rho, E$ to $V_{A} B\left(0, a_{0}\right) / c, x$ and $y$ to $L$, and $t$ to $L / V_{A}$. The parameter $a_{0}$ is the thickness of the current layer at $x=0$, and $L$ is the length of the current layer including both the diffusion and slow shock regions as shown in Figure 1. For simplicity we assume that $L$ is constant in time, but it can be allowed to vary if necessary. The variable $\eta(x)$ is the dimensionless magnetic diffusivity defined by $\eta_{e}(x) c^{2} / 4 \pi V_{A} L$ where $\eta_{e}(x)$ is the electrical resistivity. Here $\eta(x)$ is assumed to be a given function of $x$, as done in most numerical simulations of Petschek reconnection. ${ }^{2,5,28}$ It can easily be generalized, however, to be a function of the current density as well. ${ }^{13,14}$ The Lundquist number $L u$ is given simply by $1 / \eta(0)$, the inverse of the normalized diffusivity at $x=0$.

Next, following standard procedure, ${ }^{6,29}$ we expand the inflow quantities in powers of the inflow Alfvén Mach number, $M_{A}=u\left(0, a_{0}\right)=E\left(0, a_{0}\right)$. The perpendicular field and flow components $b$ and $u$ are of first order in $M_{A}$, while the parallel field and flow components $V$ and $B$ are of zeroth order. We also assume that variations in $x$ are of order unity (i.e., $\partial / \partial x \approx 1 / L$ ), while variations in $y$ are of order $1 / M_{A}$ (i.e., $\partial / \partial y \approx 1 / a_{0}$ ). These approximations reduce (3), (6), and (7) to

$$
\begin{gathered}
E=-V b+u B-\eta(x) B_{y}, \\
V_{t}+V V_{x}+u V_{y}=-p_{x}+b B_{y}, \\
\left(p+B^{2} / 2\right)_{y}=0 .
\end{gathered}
$$

Following Vasyliunas, ${ }^{16}$ Somov, ${ }^{18}$ and Titov, ${ }^{19}$ we also assume that the horizontal flow component at the edge of the

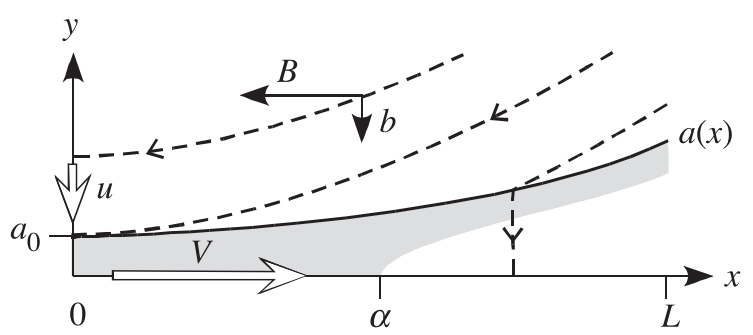

FIG. 1. Schematic diagram of the upper right quarter of the field and flow configuration. $B$ and $b$ indicate the $x$ - and $y$-components of the magnetic field (dashed curves) and $V$ and $u$ the $x$ - and $y$-components of the bulk flow (hollow arrows). The shaded area indicates the expected current density distribution. The parameter $\alpha$ is the nominal length of the diffusion region beyond which the current density is bifurcated into a pair of slow-mode shocks. $L$ is the total length of the current layer including both diffusion and shock regions. 
layer is zero. This assumption is made in anticipation of Sweet-Parker and Petschek-like solutions for which the horizontal component of the velocity in the inflow region is of second order in $M_{A} \cdot{ }^{30}$ Erkaev et al. ${ }^{6}$ have obtained numerical solutions of the reduced, time-dependent system (1)-(10). They found that this reduced system behaves in much the same way as the full system of MHD equations, as long as the flow remains laminar. In other words, Petschek-type solutions only occur if the resistivity is nonuniform.

Next, we reduce the above two-dimensional, time dependent system to a one-dimensional system by averaging the variables and equations over the half-thickness of the current layer. Upon averaging and application of the boundary condition on $V$, we obtain to order $M_{A}$

$$
\begin{gathered}
(a\langle B\rangle)_{t}-B_{a} a_{t}=E_{o}-E_{a}, \\
(a\langle b\rangle)_{t}-b_{a} a_{t}=(a\langle E\rangle)_{x}-E_{a} a_{x} \\
(a\langle V\rangle)_{t}=-\left(a\left\langle V^{2}\right\rangle\right)_{x}+B_{a} b_{o}-a B_{a} B_{a x}+\left(a\left\langle B^{2}\right\rangle\right)_{x} \\
-B_{a}(a\langle B\rangle)_{x},
\end{gathered}
$$

with

$$
\begin{gathered}
\langle E\rangle=-\langle V b\rangle+\langle u B\rangle-\eta B_{a} / a, \\
E_{a}=B_{a} u_{a}, \\
u_{a}=-(a\langle V\rangle)_{x}, \\
V_{a}=0, \\
b_{a}=b_{o}+B_{a} a_{x}-(a\langle B\rangle)_{x},
\end{gathered}
$$

where

$$
\langle V\rangle=(1 / a) \int_{0}^{a} V(x, y) d y,
$$

and the average is applied similarly to the other variables and terms that are functions of $y$. Leibniz's rule has been used to express the averages of derivatives in terms of derivatives of the averages. The subscripts with respect to $x, y$, and $t$ refer to partial derivatives, but the other subscripts do not. The variable $a(x)$ is the thickness of the current layer including both the diffusion region and the slow shock region as shown in Figure 1. Note that $a(x, t)$ is defined as occurring in the external region, immediately upstream of the current layer. Variables with the subscripts $o$ and $a$ correspond to quantities evaluated at $y=0$ (center of the layer) and $y=a$ (edge of the layer), respectively. The field component $B_{a}(x)$ is determined using an external field model (e.g., Petschek with $B_{a}=-1$ or Green-Syrovatskii with $\left.B_{a}=-\sqrt{1-x^{2}}\right)$. For simplicity, the above equations also assume that the external gas pressure is uniform so that $p(x, a)=p_{a}$ is constant in both space and time to order $M_{A}$.

To proceed further, additional assumptions need to be made about how averages of products are related to products of the averages and how center-line values at $y=0$ are related to the averaged quantities. Both Vasyliunas and Somov and Titov assume that variations of $\langle V\rangle$ in $y$ are of order $M_{A}$ or smaller. They also make the assumption that $\langle B\rangle$ is of order $M_{A}$ or smaller. While this latter assumption seems reasonable for the slow-shock region, it is less appropriate for the diffusion region where we expect $\langle B\rangle$ to be of zeroth order near $x=0$. In Sec. V, we develop a more realistic treatment for the diffusion region that does not assume that $\langle B\rangle$ is zero. As we will see in that section, the overall behavior of the solutions is not radically altered.

The assumptions that $B$ is negligible and $V$ is uniform in $y$ inside the current layer lead to

$$
\begin{array}{ll}
\langle B\rangle=0, & u_{a}=-(a\langle V\rangle)_{x}, \\
\left\langle V^{2}\right\rangle=\langle V\rangle^{2}, & b_{a}=\langle b\rangle+B_{a} a_{x}, \\
\langle V b\rangle=\langle V\rangle\langle b\rangle, & \langle u\rangle=-\langle V\rangle_{x} a / 2, \\
\langle u B\rangle=0, & b_{o}=\langle b\rangle .
\end{array}
$$

Since $B$ is negligible for $y<a$, we also have from Faraday's equation that $\partial E / \partial y=0$, so $E_{0}=\langle E\rangle$. After substituting the above relations into (11)-(18), we obtain

$$
\begin{gathered}
a_{t}=-(a V)_{x}+V b / B_{a}+\eta / a, \\
b_{t}=-(b V)_{x}-\left(B_{a} \eta / a\right)_{x}, \\
(a V)_{t}=-\left(a V^{2}\right)_{x}+B_{a} b-a B_{a} B_{a x},
\end{gathered}
$$

where the angle brackets have been dropped since we no longer need them to distinguish between averaged and unaveraged variables. Although these equations are relatively simple, they are still strongly nonlinear. The first term on the right-hand side of (19) is just the inflow velocity $u_{a}$ at the edge of the layer. Since this velocity is negative, it acts to decrease the thickness $a$ of the layer. By contrast the second and third terms act to increase $a$. The second term is due to upstream propagation of the current layer via the agency of a slow-mode wave, while the third term corresponds to upstream propagation of the current layer by diffusion.

The two terms on the right-hand side of (20) control the decay and growth of the normal magnetic field component, $b$, which accelerates the flow. The first term causes decay by advection of $b$ out of the layer, while the second causes growth by converting the tangential field $B_{a}$ into $b$ by means of diffusion (i.e., reconnection). In their publications, Malyshkin and Kulsrud place special emphasis on the importance of the latter term. ${ }^{14,15}$ As we will see in subsequent sections, it does indeed play a pivotal role in the structure and dynamics of the solutions.

The first term on the right-hand side of (21) is the acceleration of mass flux in the current layer. The second and third terms are the two forces that cause this acceleration. The $B_{a} b$ term is the Maxwell stress due to the $\mathbf{j} \times \mathbf{B}$ force within the layer, while the $a B_{a} B_{a x}$ term is a force due to a gas pressure gradient that occurs when the external magnetic field component $B_{a}$ varies in $x$. In the Green-Syrovatskii model, $B_{a}$ varies as $-\sqrt{1-x^{2}}$ from $x=0$ to $x=1$. Consequently, the balance of the total pressure across the layer leads to a decrease in the gas pressure with increasing $x$. In other words, the enhanced $B_{a}$ at the center of the sheet squeezes plasma out of the sheet. 
If $B_{a}$ is approximately uniform, as in the Petschek and Vasyliunas models, then this term is negligible.

Before considering solutions to the time-dependent system, we first consider steady-state solutions. These steady-state solutions encompass those previously obtained by Vasyliunas, ${ }^{16}$ Kulsrud, ${ }^{15}$ as well as the incompressible limit of the solutions of Somov ${ }^{17,18}$ and Titov. ${ }^{19,20}$

\section{STEADY-STATE SOLUTIONS}

In a steady-state, (19)-(21) reduce to

$$
\begin{gathered}
(a V)_{x}=V b / B_{a}+\eta / a, \\
(b V)_{x}=-\left(B_{a} \eta / a\right)_{x}, \\
\left(a V^{2}\right)_{x}=B_{a} b-a B_{a} B_{a x} .
\end{gathered}
$$

These equations prescribe $a, b, V$ as function of $x$ given the functions $B_{a}(x)$ and $\eta(x)$. For the Petschek model $B_{a}=-1$ to zeroth order in $M_{A}$, while for the Green-Syrovatskii model $B_{a}=-\sqrt{1-x^{2}}$ to zeroth order. For either model, $B_{a}$ can be iterated to obtain the first order correction once an initial solution for $b(x)$ is obtained. ${ }^{18}$ The first order correction is needed in order to obtain Petschek's result for the external Alfvén Mach number in the far upstream region. The correction procedure is briefly discussed in Appendix A. More extensive discussions can be found in several previous publications. ${ }^{1,6,18,30}$

Equations (22)-(24) correspond to a system of three firstorder ordinary differential equations (ODEs). For $B_{a}=-1$, integration of (23) immediately leads to the Ohm's law

$$
M_{A}=-b V+\eta / a,
$$

where, $M_{A}$, the reconnection rate, is a constant of integration. Substitution of this result into (22) then yields

$$
a V=M_{A}\left(x-x_{s p}\right),
$$

where $x_{s p}$ is another constant of integration. The parameter $x_{s p}$ is the location of the stagnation point where the flow $V$ is zero. For the symmetric configurations we consider here, $x_{s p}$ is zero and co-located with the neutral line. However, in asymmetric configurations this is no longer the case. ${ }^{31}$

Finally, setting $x_{s p}=0$ and using (25) and (26) to eliminate $a$ and $b$ in (24) gives an ODE for the velocity

$$
x V_{x}+V=(1 / V)-(\alpha / x)\left(\eta / \eta_{0}\right),
$$

where $\alpha=\eta_{0} / M_{A}^{2}=a_{0} / M_{A}$ is the nominal length of the diffusion region and $\eta_{0}=\eta(0)$ is the diffusivity at the neutral line. In the region $|x|<\alpha$ the diffusive electric field, $\eta j$, dominates, while in the region $|x|>\alpha$ the advective electric field, $V b$, dominates. Equation (27) is the same as the one obtained by Vasyliunas for the Petschek model (Eq. (29) in Ref. 16). It is also the same as the equation obtained by Somov and Titov in the incompressible limit (plasma $\beta \rightarrow \infty$ ) and in the limit $x \ll 1$ (Eq. (3.4.11) in Ref. 18). Numerical solutions of (27) are shown in Figure 2 for various values of the integration constant for the uniform diffusivity case, $\eta=\eta_{0}$.

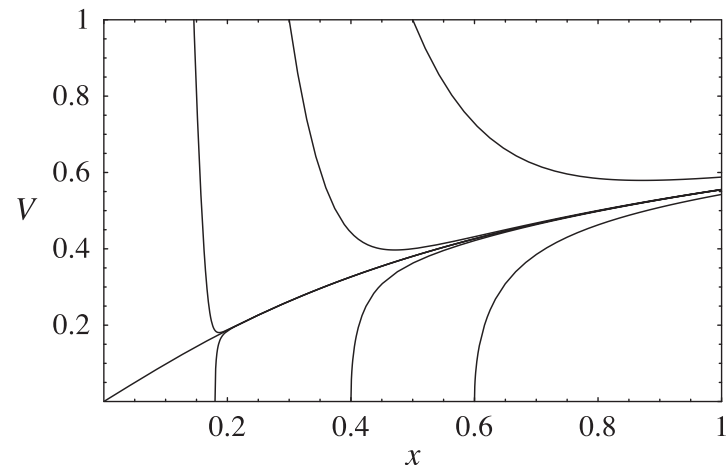

FIG. 2. Outflow velocity $V$ prescribed by (27) for different values of the integration constant. Solutions are shown for $\eta=\eta_{0}$ (uniform resistivity) and $\alpha=1$ (Sweet-Parker reconnection). Only the solution passing through the origin corresponds to a reconnection-type scenario.

From this figure, we see that there is only one value of the integration constant that gives a solution with $V(0)=0$. Thus, for symmetric solutions, there is only one constant of integration, namely the reconnection rate $M_{A}$, that remains indeterminate.

So how is the constant of integration, $M_{A}$, to be determined? Typically, a constant of integration in a steady-state ODE is set by the boundary conditions, but, as we pointed out in the introduction, Petschek reconnection is undriven. Consequently, $M_{A}$ cannot be specified in this way. If $M_{A}$ is not set by the boundary conditions, perhaps it is set by the initial state of the time-dependent system. The different steady-state solutions would then represent different choices for the initial conditions. There is, however, a problem with this interpretation. As we will discuss in Sec. IV, steadystate solutions of the time dependent system (19)-(21) are structurally unstable if both $\eta$ and $B_{a}$ are uniform. As shown in Appendix B, these unstable solutions have an essential singularity at $x=0$. Stable, nonsingular solutions appear to be possible only when either $\eta$ or $B_{a}$ is nonuniform.

We can distinguish between stable and unstable solutions by considering a power series expansion of (27) around the X-line. Let us consider the case where $B_{a}$ is uniform and equal to -1 , but $\eta$ is nonuniform, then

$$
\begin{gathered}
V(x)=V_{1} x+V_{3} x^{3}+\cdots=\sum_{n=0}^{\infty} V_{2 n+1} x^{2 n+1}, \\
\eta(x)=\eta_{0}+\eta_{2} x^{2}+\cdots=\sum_{n=0}^{\infty} \eta_{2 n} x^{2 n} .
\end{gathered}
$$

Upon substitution into (27), we obtain to lowest order

$$
x\left(1-\alpha V_{1}\right)=0,
$$

and to higher orders

$$
\begin{aligned}
\sum_{n=0}^{\infty}[ & 2 \sum_{i=0}^{n}(i+1) V_{2 i+1} V_{2(n-i)+1} \\
& \left.+\frac{\alpha}{\eta_{0}} \sum_{i=0}^{n+1} \eta_{2 i} V_{2(n-i)+3}\right] x^{2 n+3}=0 .
\end{aligned}
$$


Equation (31) gives a recursive relation for the series coefficients.

Generally, the power series (28) constitutes an asymptotic expansion that diverges for any $x>0$ as $n$ goes to $\infty$ (see Appendix B). There are, however, special cases that have the property that for a particular value of $\alpha$ (and, hence, a particular value of $M_{A}$ ) the series (28) appears to be convergent, rather than divergent. Consider the specific case of the diffusivity with the Gaussian profile

$$
\eta(x)=\eta_{0} \exp \left(-x^{2} / l_{g}^{2}\right)=\eta_{0}\left(1-x^{2} / l_{g}^{2}+x^{4} / 2 l_{g}^{4}-\cdots\right),
$$

that is often used in numerical simulations to obtain Petschek-like solutions. ${ }^{2}$ Substituting into (31), we obtain for the first four coefficients

$$
\begin{aligned}
V_{1} & =1 / \alpha, \quad V_{3}=\left(-2+\alpha^{2} / l_{g}^{2}\right) / \alpha^{3}, \\
V_{5} & =\left(24-16 \alpha^{2} / l_{g}^{2}+\alpha^{4} / l_{g}^{4}\right) / 2 \alpha^{5}, \\
V_{7} & =\left(-672+552 \alpha^{2} / l_{g}^{2}-90 \alpha^{4} / l_{g}^{4}+\alpha^{6} / l_{g}^{6}\right) / 6 \alpha^{7} .
\end{aligned}
$$

As $n$ approaches $\infty, V_{n}$ approaches $+\infty$ or $-\infty$ for all values of $\alpha$ except one. This unique value of $\alpha$ is determined by the condition

$$
\lim _{n \rightarrow \infty}\left|V_{2 n+3}(\alpha)\right|=0
$$

which is a necessary (but not sufficient) condition for the convergence of the power series (28). If the power series converges, then the solution $V(x)$ is analytic at $x=0$. The special value of $\alpha$ can be approximately determined by setting anyone of the coefficients, $V_{n}$, to zero for $n>1$. Increasingly larger values of $n$ give increasingly better approximations.

The lowest order approximation is obtained by setting $V_{3}=0$. This gives

$$
\begin{aligned}
\alpha & =\sqrt{2} l_{g}=1.41421 \lg , \\
M_{A} & =\sqrt{\eta_{0} / \alpha}=2^{-1 / 4} \eta_{0}^{1 / 2} l_{g}^{-1 / 2} .
\end{aligned}
$$

For the next order approximation, we set $V_{5}=0$. This gives two positive roots, namely

$$
\alpha=\sqrt{2(4 \pm \sqrt{10})} l_{g} .
$$

When there are multiple roots, the root with the smallest positive value yields an approximation for the $\alpha$ value of the nonsingular solution. Table I show the values of $\alpha$ obtained by setting the next three orders of $V_{n}$ to zero. The table value for $V_{11}=0$ agrees with the value from the numerical solution of the time-dependent nozzle equations (last line in table) to six significant figures. This good agreement, however, does not mean that we have calculated the reconnection rate to a high accuracy. The one-dimensional nozzle equations are highly idealized, and they are unlikely to be accurate to more than $10 \%$ to $20 \%$. $^{32}$

Whether or not a nonsingular solution exists depends on the functional form of $\eta(x)$. For the uniform case $\left(l_{g}=\infty\right)$,
TABLE I. Diffusion region length $\alpha$ for different levels of approximation.

\begin{tabular}{lcc}
\hline \hline Level of approximation & $\begin{array}{c}\text { Analytical expression } \\
\text { for } \alpha / l_{g}\end{array}$ & $\begin{array}{c}\text { Decimal value } \\
\text { for } \alpha / l_{g}\end{array}$ \\
\hline$V_{3}=0$ & $\sqrt{2}$ & 1.41421 \\
$V_{5}=0$ & $\sqrt{2(4-\sqrt{10})}$ & 1.29439 \\
$V_{7}=0$ & Solution of cubic & 1.28709 \\
$V_{9}=0$ & Solution of quartic & 1.28675 \\
$V_{11}=0$ & No exact form & 1.28674 \\
Numerical solution & Not applicable & 1.28674 \\
of PDEs as $t \rightarrow \infty$ & & \\
\hline \hline
\end{tabular}

all solutions have an essential singularity at $x=0$ (see Appendix $\mathrm{B}$ for a formal proof). The choice $\eta(x)=$ $\eta_{0} \exp \left(-x^{4} / l_{g}^{4}\right)$ also fails to yield a nonsingular solution for any finite value of $\alpha$. Although this form is nonuniform and highly localized, it has a very flat profile near $x=0$, and it fails to produce a steady-state when it is used in the timedependent system. In order for a nonsingular solution to exist the Taylor expansion of $\eta(x)$ around $x=0$ must have a negative quadratic term. If $\eta(x)$ has a variation that is purely quadratic, i.e., $\eta(x)=\eta_{0}\left(1-x^{2} / l_{g}^{2}\right)$, then (27) has the exact solution, $V=x / \alpha$ when $\alpha=\sqrt{2}$. This solution is the only nonsingular solution. It corresponds to a current sheet which has uniform thickness, $a_{0}$, and a linear variation of the transverse field $b(x)$.

Figure 3 plots $V(x), a(x)$, and $b(x)$ for the nonsingular solution for $l_{g}=0.1$ with $\eta_{0}=10^{-4}$ (i.e., $L u=10^{4}$ ). The solution is Petschek-like with a short diffusion region $(\alpha=0.129)$ corresponding to a reconnection rate of $M_{A}=0.0279$. In the downstream region $V(x) \rightarrow 1$, (the ambient Alfvén speed), $a(x) \rightarrow M_{A} x$, and $b(x) \rightarrow-M_{A}$ as required for Petschek's model. It is difficult visually to distinguish a nonsingular reconnection solution, such as that shown in Figure 3, from a singular one, such as that shown in Figure 2 for the curve with $V(0)=0$. The only noticeable difference is that a nonsingular solution appears somewhat smoother than a singular one. This difference, however, is very subtle, because it depends on the behavior of the higher order derivatives at $x=0$.

In their two-dimensional MHD simulations Baty et al. ${ }^{28}$ found that simply localizing $\eta(x)$ around $x=0$ is not

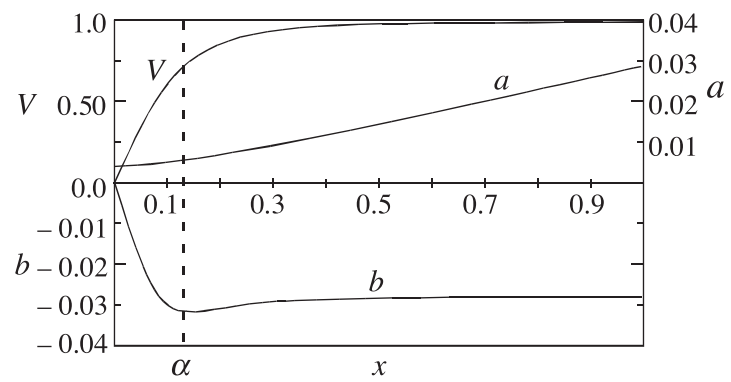

FIG. 3. The outflow velocity $V$, current layer thickness $a$, and transverse field component $b$ as functions of the distance, $x$, along the current layer. The neutral line is at $x=0$ and the tip of the current layer (including slow shocks) is at $x=1$. The vertical dashed line at $x=\alpha$ indicates the tip of the diffusion region. This particular solution is the only nonsingular solution of the nozzle Equation (27) when the magnetic diffusivity has a Gaussian profile with scale length $l_{g}=0.1$. 
sufficient to guarantee a Petschek-like solution. It is also necessary that the second derivative of $\eta$ be negative at $x=0$. A $\eta$ profile that is too flat or first increases before decreasing leads to a configuration that never stabilizes in the twodimensional simulations. Our singularity analysis also predicts that a stable solution will not exist in such cases.

If the diffusivity is uniform or varies too slowly, then the variation in the external field component $B_{a}(x)$ becomes an important factor in stabilizing the configuration. While such variation is ignored in Vasyliunas's analysis, it is included in the Somov-Titov analysis. They use the GreenSyrovatskii formula $B_{a}=-\sqrt{1-x^{2}}$ for an infinitely thin current sheet of length one in our normalized units. This formula for $B_{a}$ does not hold all the way to $x=1$ since the equations become singular there. The range of validity is approximately $|x| \leq 1-M_{A}$. When $B_{a}=-\sqrt{1-x^{2}}$, Eq. (27) is replaced by

$$
\begin{aligned}
\sin ^{-1} x & V_{x}+V / \sqrt{1-x^{2}} \\
& =\left(\sqrt{1-x^{2}}+x \sin ^{-1} x\right) / V-\left(\alpha \eta / \eta_{0} \sin ^{-1} x\right)\left(1-x^{2}\right) .
\end{aligned}
$$

The above equation is the same as the equation obtained by Somov and Titov for uniform resistivity in the limit that the plasma $\beta \rightarrow \infty$ (the incompressible limit). Expanding yields $V_{1}=1 / \alpha$ and $V_{3}=\left(-6+5 \alpha^{2}\right) / 3 \alpha^{3}$ for the first two terms. As before, the series does not converge except for one particular value of $\alpha$. Setting $V_{3}=0$ gives a rough estimate for this value of $\alpha \approx \sqrt{6 / 5} \approx 1.09$. Setting $V_{13}=$ gives the more precise value of 1.049092 . The requirement that the solution be nonsingular predicts that for uniform diffusivity the only stable solution for the Green-Syrovatskii $B_{a}$ profile is the Sweet-Parker one which has $\alpha \approx 1$.

For locally enhanced, nonuniform resistivity, setting $V_{3}=0$ in the power series expansion of (32) yields the approximate formula $\alpha \approx \sqrt{6 /\left(5-3 \eta_{2} / \eta_{0}\right)}$ for the length of the diffusion region. In the case of the Gaussian profile, this corresponds to a reconnection rate of

$$
M_{A} \approx \eta_{0}^{1 / 2}\left[\left(5 l_{g}^{2}+3\right) / 6 l_{g}^{2}\right]^{1 / 4} .
$$

The Green-Syrovatskii result for uniform diffusivity is recovered when $l_{g} \rightarrow \infty$, and the $B_{a}=-1$ result for the Gaussian profile is recovered when $l_{g} \ll 1$ (top line, Table I).

The analysis of Malyshkin and Kulsrud is equivalent to a first order expansion of (22)-(24) around $x=0$. To first order, these equations are

$$
\begin{gathered}
a_{0} V_{1}=\eta_{0} / a_{0} \\
b_{1} V_{1} x=a_{2} \eta_{0} B_{a 0} x / a_{0}^{2}-B_{a 0} \eta_{0}^{\prime \prime} x / 2 a_{0}-B_{a 0}^{\prime \prime} \eta_{0} x / 2 a_{0} \\
2 a_{0} V_{1}^{2} x=B_{a 0} b_{1} x-a_{0} B_{a 0} B_{a 0}^{\prime \prime} x
\end{gathered}
$$

where

$$
\begin{aligned}
a(x) & \approx a_{0}+a_{2} x^{2}, \\
B_{a}(x) & \approx B_{a 0}+B_{a 0}^{\prime \prime} x^{2} / 2, \\
\eta(x) & \approx \eta_{0}+\eta_{0}^{\prime \prime} x^{2} / 2 .
\end{aligned}
$$

These are the same equations used by Malyshkin and Kulsrud except for the first term on the right-hand side of (35) that contains the factor $a_{2}$. Malyshkin and Kulsrud assume that $a(x)$ is uniform in the diffusion region, and, consequently, their equation lacks this term. Since $B_{a 0}=-1$ and $a_{0}=M_{A} \alpha$, we can rewrite (34) and (36) as

$$
\begin{gathered}
V_{1}=1 / \alpha, \\
b_{1}=-M_{A}\left(2 / \alpha-\alpha B_{a 0}^{\prime \prime}\right) .
\end{gathered}
$$

Substituting these into (35) yields

$$
M_{A}^{4}=3 \eta_{0}^{2} B_{a 0}^{\prime \prime} / 4-\eta_{0} \eta_{0}^{\prime \prime} / 4+a_{2} \eta_{0} M_{A} / 2
$$

Setting $a_{2}=0$ gives Malyshkin and Kulsrud's formula for the reconnection rate (Eq. (11) in Ref. 14). If we now use the Green-Syrovatskii model to evaluate $B_{a}$, and assume that $\eta$ is uniform, then $B_{a 0}^{\prime \prime}=1, \eta_{0}^{\prime \prime}=0$, and

$$
M_{A}=\eta_{0}^{1 / 2}(3 / 4)^{1 / 4} \approx 0.93 \mathrm{Lu}^{-1 / 2},
$$

which is the slow Sweet-Parker rate. This result is why Malyshkin and Kulsrud conclude that a fast, Petschek-type solution cannot occur if $\eta$ is uniform. This conclusion, however, only follows if $a_{2}$ is set to zero. In the analyses of Vasyliunas and Somov and Titov, $a_{2}$ is not set to zero. Instead, its value is given by (39) as a function of an indeterminate $M_{A}$.

Malyshkin and Kulsrud do not explain why they omit the $a_{2}$ term in (39), but a justification for doing so can be found from the requirement that the solution should be nonsingular at $x=0$. The second order term in the expansion for $a$ generates a first order term when it is substituted into the right-hand side of (23). This property is closely related to the presence of a singularity at $x=0$. If one attempts to solve for $a_{2}$ by carrying out the expansion to the next order, then $a_{4}$ appears, and so on for higher orders. In the singular solutions, the terms containing the next higher order coefficients can never be ignored. However, if the solution is analytic at $x=0$, then the terms containing the next higher order coefficients will become smaller as the order rank increases. Setting $a_{2}=0$, as Malyshkin and Kulsrud do, or $V_{3}=0$ as in Table I, provides a lowest order estimate for the reconnection rate of the nonsingular solution.

\section{TIME-DEPENDENT SOLUTIONS}

The one-dimensional system is much simpler mathematically than the two-dimensional, resistive MHD system that it approximates. Much of the physical complexity of the two-dimensional system has therefore been lost. Nevertheless, the reduced system still retains some interesting behavior that appears to be similar to that observed in laminar two-dimensional, resistive MHD simulations carried out at Lundquist numbers below $10^{4}$. Although (19)-(21) look relatively simple, they are highly nonlinear and mathematically equivalent to a third order partial differential equation (PDE). Since they cannot be solved analytically except for a few special cases, we use a numerical method. In order 
to solve the system, we first need to determine the number of initial and boundary conditions that are required. As shown in Appendix C, the system (19)-(21) is purely hyperbolic with a single characteristic speed, namely the flow speed, $V$. Consequently, the number of boundary conditions required for a solution depends only upon the direction of the flow at the outflow boundaries. For the numerical solutions we locate our boundaries at $x= \pm 0.9$, in order to avoid the null regions at $x= \pm 1$ for those cases that use the GreenSyrovatskii model $B_{a}=-\sqrt{1-x^{2}}$. At these locations the flow is always outward, and information cannot propagate from the outside region back into the numerical domain. Therefore, no boundary conditions can be imposed at $x= \pm 0.9$ unless the flow there reverses direction. All that is required to obtain a unique solution are initial conditions for $a, b$, and $V$ at $t=0$. In the time-dependent system, $M_{A}$, the undetermined constant of integration in the steady-state solution becomes a function of time. Its initial value is determined by the choice of the initial state, so its value is no longer indeterminate if the initial state of the system is known.

To solve (19)-(21), we use the "method-of-lines" as implemented in MATHEMATICA. Typically, we use an 8th order difference scheme with grid resolutions in the range from 100 to 200 grid points. The "method-of-lines" has the advantage of being a versatile solver for parabolic and hyperbolic PDEs. However, it has the disadvantage of being prone to numerical instability if steep gradients are present in the solution. Therefore, to avoid the development of a numerical instability we use initial conditions that are relatively smooth.

Figure 4 shows a numerical solution of the time dependent equations for a case with $\eta=\eta_{0} \exp \left(-x^{2} / l_{g}^{2}\right), \eta_{0}=10^{-4}$, and $B_{a}=-1$. The initial conditions for this case are $a=0.0046, V=x /\left(\sqrt{2} l_{g}\right)$, and $b=-a_{0} x / l_{g}$. These conditions do not correspond to a steady state, so the initial state immediately starts to evolve. After a few Alfvén scale times, the configuration settles into a steady-state that matches the unique, nonsingular solution of the steady-state equation, (27), to within an accuracy of six significant figures. All other choices for the initial condition that we have tested give the same result, including those based on the singular, steadystate solutions of (27). Therefore, we infer that the nonsingular solution acts as an attractor of the dynamical system towards which all solutions evolve.

Figure 5 shows the reconnection rate as a function of time for two different initial conditions based on singular steady-states. The curve labeled "too fast" corresponds to a steady-state Petschek-like solution with an initial reconnection rate of $M_{A}=0.05$. This value is almost twice the final steady-state rate of $M_{A}=0.0279$ which is reached by $t=1.5$. This final value is exactly the one corresponding to the nonsingular solution of the steady-state equation. The curve labeled "too slow" corresponds to a steady-state Petschek-like solution with an initial value of $M_{A}=0.023$. In both cases the initial state is unstable, and it rapidly evolves towards the nonsingular steady-state solution with $M_{A}=0.0279$ (dashed line).

If both the diffusivity, $\eta$, and the external field, $B_{a}$, are uniform, then all steady-state solutions are singular at $x=0$. These cases, which correspond to the original Petschek model, have the behavior shown in Figure 6. As before, the initial steady-state is unstable, but the configuration no longer rapidly evolves to a new stable configuration. Instead, the reconnection rate, $M_{A}$, continually decays towards zero, and the length of the diffusion region increases linearly with time. This long-term behavior corresponds to a self-similar solution of (19)-(21). When $\eta$ and $B_{a}$ are both uniform these equations no longer have an inherent scale length, so a selfsimilar solution becomes possible. The self-similar variables are $a_{*}=a t^{-1 / 2}, b_{*}=b t^{1 / 2}$, and $\xi=x / t$, which upon substitution in (19)-(21) yield the ODEs

$$
\begin{gathered}
\frac{1}{2} a_{*}-\xi a_{* \xi}=-\left(a_{*} V\right)_{\xi}-b_{*} V+\eta_{0} / a_{*}, \\
-\frac{1}{2} b_{*}-\xi b_{* \xi}=-\left(b_{*} V\right)_{\xi}+\left(\eta_{0} / a_{*}\right)_{\xi}, \\
\frac{1}{2} a_{*} V-\xi\left(a_{*} V\right)_{\xi}=-\left(a_{*} V^{2}\right)_{\xi}-b_{*} .
\end{gathered}
$$

Expanding these equations around $\xi=0$ yields the first order solution

$$
a(x)=a_{* 0} t^{1 / 2}, \quad b(x)=b_{* 1} x t^{-3 / 2}, \quad V(x)=V_{1} x t^{-1}
$$

that describes the long-term evolution towards a simple, uniform current layer with no transverse field or flow. As the transverse field, $b$, declines with time, the flow, $V$, that it accelerates also declines. All that remains at large times is a uniform current layer widening at the diffusive rate of $t^{1 / 2}$.

The nature of the instability manifest in Figure 6 is closely linked to the presence of the essential singularity in the initial states. The singularity cannot be precisely represented in any finite difference scheme because it would
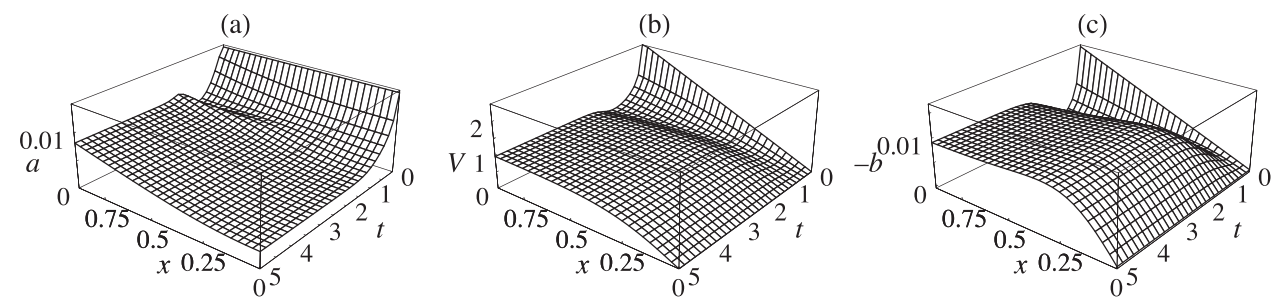

FIG. 4. This figure shows the transition from a nonequilibrium initial state to the nonsingular steady state for a uniform external field $\left(B_{a}=-1\right)$ and a Gaussian diffusivity profile with the scale length $l_{g}=0.25$ in normalized units. Panels (a), (b), and (c) show the layer thickness, $a$, the outflow velocity, $V$, and the transverse magnetic field, $b$, respectively, as functions of the distance, $x$, and the time, $t$. By $t=5$ the numerical solution matches the nonsingular steadystate solution to an accuracy of $10^{-6}$. 


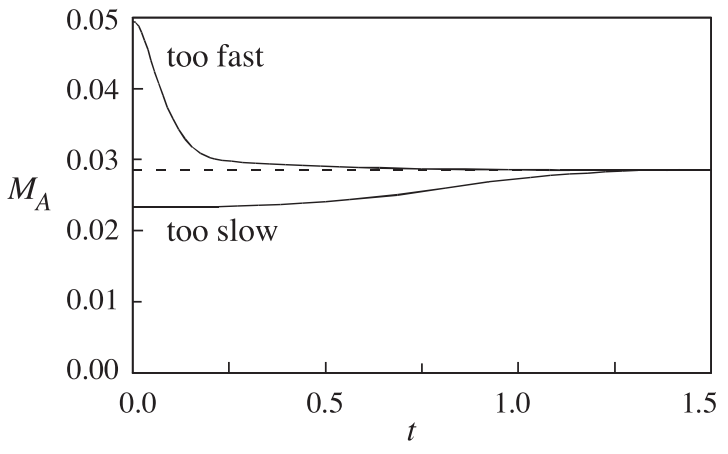

FIG. 5. Evolution of the reconnection rate, $M_{A}$, for two different singular, steady-state solutions. For these solutions, the diffusivity is nonuniform and has a Gaussian profile with a scale length $l_{g}=0.1$. The time $t$ is normalized to the Alfvén scale time. Both solutions rapidly evolve towards the reconnection rate of $M_{A}=0.0279$ of the nonsingular steady-state solution (dashed line). Here, $\eta_{0}=10^{-4}$ and $B_{a}=-1$.

require an infinitely fine grid to do so. Since there are no nonsingular equilibria for the uniform $\eta$ and $B_{a}$ case, the smoothed initial state cannot be an exact, steady-state equilibrium. Therefore, it must evolve, but there are two possibilities. It evolves so as to become as close to the singular state as the grid resolution will allow (as occurs when a compressive wave steepens into shock), or it evolves away from the singular state. Which outcome occurs depends on the last term on the right-hand side of (20). This is the term that continually creates the transverse field, $b$, in the layer, and in a true equilibrium it should exactly balance the first term on the right-hand side of (20), which depletes the transverse field by advecting it out of the layer.

The ability of the last term in (20) to balance advection depends critically on the existence of the singularity at $x=0$. The link between this term and the singularity is apparent if we expand the time-dependent (19)-(21) in powers of $x$. To lowest order, this yields

$$
\begin{gathered}
a_{0 t}=-a_{0} V_{1}+\eta / a_{0}, \\
b_{1 t}=-2 b_{1} V_{1}-2 \eta a_{2} / a_{0}^{2}, \\
\left(a_{0} V_{1}\right)_{t}=-2 a_{0} V_{1}^{2}-b_{1},
\end{gathered}
$$

where $a(x, t)=a_{0}(t)+a_{2}(t) x^{2}+\cdots, b(x, t)=b_{1}(t) x+b_{3}(t) x^{3}$ $+\cdots, V(x, t)=V_{1}(t) x+V_{3}(t) x^{3}+\cdots$. The last term in (45)

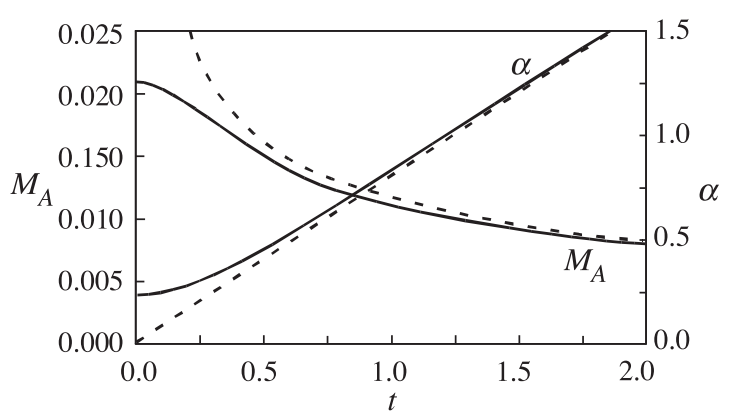

FIG. 6. Evolution of the reconnection rate, $M_{A}$, and the diffusion region length, $\alpha$, for a steady-state solution with a uniform diffusivity profile and a uniform $B_{a}$. The unstable initial configuration does not reach a new equilibrium. Instead, it approaches the long-term decay behavior of the self-similar solution (dashed lines). Here, $\eta_{0}=10^{-4}$. corresponds to the last term in (20). Note that it contains the second order coefficient $a_{2}$. Were it not for the presence of the unknown $a_{2}$, (44)-(46) would be a closed system, and $a_{0}, b_{1}$, and $V_{1}$ could be determined as functions of time. If we set $a_{2}=0$ as Malyshkin and Kulsrud do, then (45) requires that the magnitude of $b_{1}$ decreases with time as shown in Figure 7. If we go to the next higher order in the expansion, we can obtain equations for $a_{2}, b_{3}$, and $V_{3}$, but again the last term in (45) leads to the introduction of a new unknown, namely $a_{4}$. Higher orders proceed in a similar fashion. If at any order $n$ we throw away the term with the unknown coefficient $a_{n+1}$ in the last term of the equation for $b_{n t}$, then the transverse field $b$ will decay. Decay can only be prevented by increasing the truncation order all the way to $\infty$. This behavior is linked to the presence of the essential singularity at $x=0$. Truncation of the infinite series eliminates the singularity and leads to the decay of the transverse field needed to accelerate the flow. As the flow declines, the thickness, $a$, of the layer becomes increasingly uniform, diffusing outwards at a rate proportional to $t^{1 / 2}$.

Figure 7 also shows the result obtained when $a_{10}$ is set to zero. The solution is obtained by numerically solving the 15 coupled equations obtained by expanding (19)-(21) to order 8 and then dropping the term containing the $a_{10}$ coefficient. The time for the decay to become manifest is approximately proportional to $\ln n$, so increasing the truncation order from 10 to 100 only increases this time by about a factor of two. At very large times, the decay is given by the self-similar solution following equation (43).

The existence of a singularity in the steady-state solutions is related to the flow of information in the solutions. As discussed in Appendix C, the averaged equations are purely hyperbolic and have the single characteristic speed $V$. Therefore, information about the initial conditions propagates into the rest of the solution along the characteristic paths defined by $\mathrm{d} x / \mathrm{d} t=V(x, t)$. For a steady state, $V(x) \approx x / \alpha$ near the $\mathrm{X}$-line, and the characteristics paths are approximately $x=x_{i} \exp (t / \alpha)$ where $x_{i}$ is the initial location of a particular

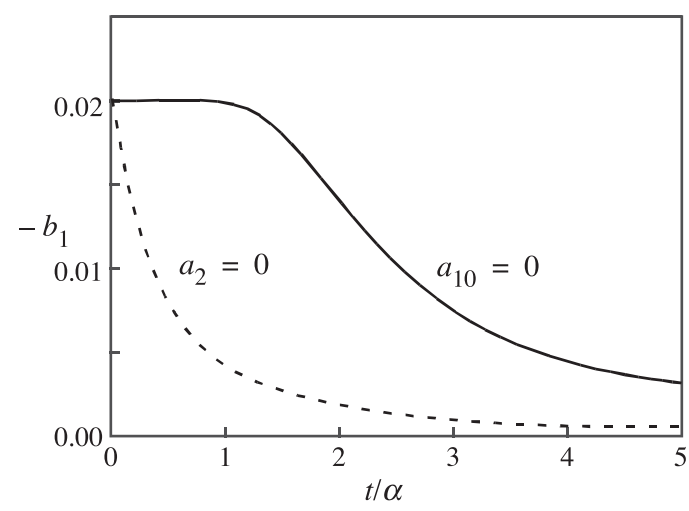

FIG. 7. These curves show the decay of the transverse magnetic field that occurs when a singular, steady-state equilibrium with uniform resistivity is smoothed. The parameter $b_{1}$ is the spatial gradient of the transverse magnetic field at $x=0$. The dashed curve labeled $a_{2}=0$ corresponds to the case considered by Malyshkin and Kulsrud where the infinite series expansion for the solution is truncated by assuming that $\partial^{2} a / \partial x^{2}=0$ at $x=0$. The solid curve labeled $a_{10}=0$ shows the decay when the initial state is truncated at the 10 th order by setting $\partial^{10} a / \partial x^{10}=0$ at $x=0$. For both cases, $\eta_{0}=10^{-4}$ and $B_{a}=-1$. 
characteristic (see Figure 8). As $t \rightarrow \infty$, only the straight line characteristic at $x_{i}=0$ links the final state to the initial state. All other information about the initial conditions has been advected into the far downstream regions. Since both $b(0,0)$ and $V(0,0)$ are zero at $x_{i}=0$, one might reasonably expect that the solution at large times should be indistinguishable from that of a simple current sheet with no flow, no transverse field, and a uniform thickness that increases as $\sqrt{2 \eta t}$. This is, in fact, the behavior observed for nonsingular initial conditions. When the initial conditions contain an essential singularity at $x=0$, the situation changes. The singularity has an infinitesimally small length scale associated with its infinite order derivative, and this scale cannot be removed by advection. This property allows singular steady-state solutions to retain $M_{A}$ as a free parameter.

In cases where either $\eta$ or $B_{a}$ is an imposed nonuniform function of $x$, nonsingular solutions may exist. In such cases, the variation of $\eta$ or $B_{a}$ acts a permanent source of transverse field $b$ that cannot be swept away by advection [cf. (20)]. The imposed variation must be such that it generates a transverse field consistent with reconnection.

\section{IMPROVED DIFFUSION REGION MODEL}

Both Vasyliunas ${ }^{16}$ and Somov ${ }^{18}$ and Titov ${ }^{19}$ neglect the parallel component of the magnetic field within current layer by assuming that $\langle B\rangle$ is of order $M_{A}$ or smaller. They also assume that the average of a product is equal to the product of its averages. For example, that $\left\langle V^{2}\right\rangle=\langle V\rangle^{2}$. In the slowshock region of the original Petschek model $\langle B\rangle$ is zero and $V$ is uniform in $y$, so that both assumptions are reasonable. Within the diffusion region, however, $\langle B\rangle$ is not negligible, and $V$ is not uniform in $y$. Since the properties of the diffusion region play a significant role in the dynamics of the current layer, it is important to assess the sensitivity of the results obtained in Secs. II-IV to the assumption that $\langle B\rangle$ is negligible. One way to do this, in the absence of a selfconsistent, two-dimensional solution, is to consider alternative assumptions about the structure of the interior of the diffusion region and then determine how these alternative assumptions affect the previous results.

Neither Somov and Titov nor Vasyliunas prescribes any specific functional form for the interior field and flow. They only prescribe the properties of the averaged quantities.

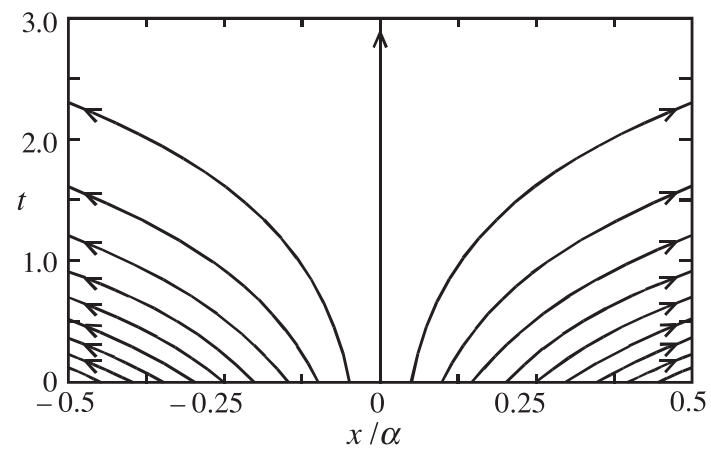

FIG. 8. Characteristic paths in the center of the diffusion region for uniform $\eta$ and $B_{a}$. Only the single characteristic at $x=0$ connects the steady-state solution at $t=\infty$ with its initial conditions.
Nevertheless, it is convenient to think of their results as corresponding to an interior model with $B(x, y, t) \approx 0$ and $V(x, y, t) \approx V(x, t)$. This model is consistent with their assumptions, but there are many different choices one could make that would lead to exactly the same equations for the averaged quantities. Once $B$ and $V$ are given, then the functional forms of $b$ and $u$ automatically follow from the solenoidal conditions $\nabla \cdot \mathbf{B}=0$ and $\nabla \cdot \mathbf{V}=0$. The simplest alternative to setting $B$ to zero and $V$ uniform in $y$ is to assume that $B$ varies linearly and $V$ varies quadratically as

$$
\begin{gathered}
B \approx-y / a, \\
V \approx V_{o}\left(1-y^{2} / a^{2}\right),
\end{gathered}
$$

where $V_{o}=V(x, 0, t)$ and, for simplicity, we have used $B_{a}=-1$. Although these are still rudimentary approximations for $B$ and $V$, they are not quite as rudimentary as simply setting $B$ to zero and $V$ uniform. If $B$ really were zero, then there would be no current density and no $\mathbf{j} \times \mathbf{B}$ force to accelerate the flow in the diffusion region. Having $B$ vary linearly with $y$ does produce a current density, but one that is uniform in $y$ at $x=0$. The actual current density, however, must vary with $y$ in order to be internally consistent with Ohm's law.

From (47) and (48), it follows that

$$
\begin{gathered}
b \approx b_{o}-y^{2} a_{x} / 2 a^{2} \\
u \approx-V_{o x} y+\left[a V_{o x}-2 V_{o} a_{x}\right] y^{3} / 3 a^{3},
\end{gathered}
$$

where $b_{o}=b(x, 0, t)$. Upon averaging, these expressions lead to

$$
\begin{aligned}
& \langle B\rangle=-1 / 2, \\
& \left\langle V^{2}\right\rangle=(6 / 5)\langle V\rangle^{2} \text {, } \\
& \left\langle B^{2}\right\rangle=1 / 3 \text {, } \\
& \langle V b\rangle=\langle V\rangle\langle b\rangle+\langle V\rangle a_{x} / 15, \quad b_{a}=\langle b\rangle-a_{x} / 3, \\
& \langle u B\rangle=2\langle V\rangle_{x} a / 5+\langle V\rangle a_{x} / 5, \quad b_{o}=\langle b\rangle+a_{x} / 6
\end{aligned}
$$

The above expressions provide a more plausible model for the diffusion region, but a less plausible one for the slowshock region. From Faraday's equation, the average electric field is just

$$
\langle E\rangle=E_{o}-a_{t} / 6
$$

By using Faraday's equation to calculate $\langle E\rangle$, we ensure that electric field is uniform in $x$ in a steady-state. Substituting these expressions into (11)-(18) yields the alternative timedependent equations

$$
\begin{gathered}
a_{t} / 3=-3(a V)_{x} / 5-4 V a_{x} / 15-V b+\eta / a, \\
b_{t}=\left[-V b+2(a V)_{x} / 5-4 V a_{x} / 15+\eta / a\right]_{x}, \\
(a V)_{t}=-6\left(V^{2} a\right)_{x} / 5-b-a_{x} / 3 .
\end{gathered}
$$

The last term in (51) introduces a new physical effect that is absent in the previous system of equations. This new term decelerates the flow, while previously all the force terms acted to accelerate it. Half of the new term is due to an 
adjustment in the strength of the $\mathbf{j} \times \mathbf{b}$ force, but the other half is due to an inverse pressure gradient. The inverse pressure gradient is caused by the decrease of the parallel field component $B$ with $x$. Total pressure balance across the current layer means that the gas pressure must increase if the magnetic pressure decreases. This average gas pressure force is $\langle\partial p / \partial x\rangle=-a_{x} /(6 a)$.

In a steady-state, (49)-(51) imply

$$
x V_{x}\left[1-1 /\left(18 V^{2}\right)\right]+V=4 /(9 V)-5 \alpha \eta /\left(6 x \eta_{0}\right) .
$$

The factor of $\left[1-1 /\left(18 V^{2}\right)\right]$ in $(52)$ is a consequence of the new retarding force in (51). This factor leads to a critical point in the solutions as shown in Figure 9. The critical point occurs at $x_{c}=5 \alpha /(7 \sqrt{2})$ and $V_{c}=1 /(3 \sqrt{2})$. Only the solution that passes through the critical point has both $V(0)=0$ and a finite speed as $x$ goes to infinity. The other solutions either die away to zero or are unphysical because they are double valued or do not have $V(0)=0$. As $x$ increases, the solution passing through the critical point asymptotes to an average velocity of $2 / 3$ times the Alfvén speed. This value corresponds to a center line velocity, $V_{o}=V(x, 0)$, that is equal to the Alfvén speed since $V_{o}=3 / 2\langle V\rangle$. The critical point is analogous to the one that occurs in Parker's solar wind model, except that here it corresponds to a flow that exceeds an escape velocity rather than a supersonic transition. The retarding effect of the pressure force near the tip of the diffusion region plays a similar role to the retarding effect of gravity in Parker's solar wind solution.

For a nonuniform resistivity, stable solutions can be found by using the same technique as before, namely determining the value of $\alpha$ that removes the essential singularity at $x=0$. The lowest order coefficient is now

$$
V_{1}=3 /(5 \alpha),
$$

while the next order is

$$
V_{3}=-27\left(36+25 \alpha^{2} \eta_{2} / \eta_{0}\right) /\left(875 \alpha^{3}\right)
$$

Setting $V_{3}$ to zero gives the lowest order approximation for the reconnection rate of

$$
\alpha=(6 / 5) \sqrt{-\eta_{0} / \eta_{2}} .
$$

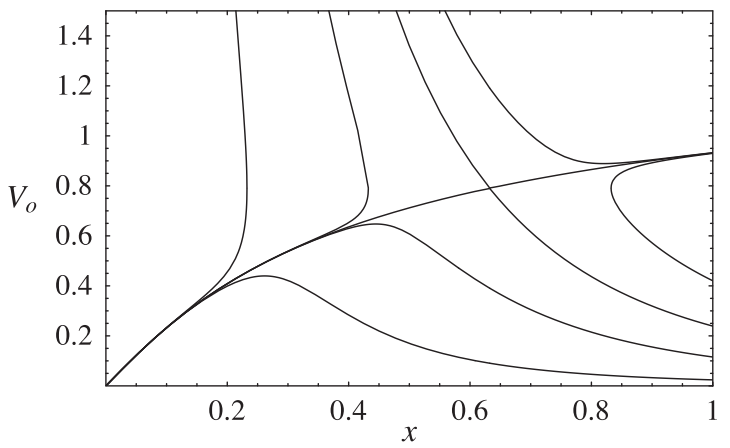

FIG. 9. Plot of solutions to (52) assuming uniform resistivity. Solutions are shown for $\alpha=1$. The critical point for the flow to escape at the Alfvén speed is located at $x=0.505$ and $V=0.236$. The solution passing through the critical point is structurally unstable.
For the Gaussian profile $\eta_{2}=-\eta_{0} / l_{g}^{2}$, so the length of the diffusion region is approximately $\alpha=1.2 l_{g}$. A more accurate approximation is $\alpha=1.1109 l_{g}$, which is obtained by setting $V_{13}=0$ in the expansion for $V$. For $l_{g}=0.1$ and $\eta_{0}=10^{-4}$, the corresponding value of $M_{A}$ is 0.0300 . Direct numerical solution of the time-dependent system (49)-(51) gives exactly the same result. This value of $M_{A}$ differs only by $7.1 \%$ from the simpler model in Secs. II-IV.

\section{CONCLUSIONS}

We have shown that the previous solutions of Petscheklike reconnection obtained by Vasyliunas ${ }^{16}$ are structurally unstable if the magnetic diffusivity is uniform. Related solutions obtained by Somov ${ }^{17,18}$ and Titov ${ }^{19,20}$ that are based on the configuration considered by Green ${ }^{22}$ and Syrovatskii ${ }^{23}$ are also structurally unstable, except for the special case of Sweet-Parker reconnection. The structural instability is associated with the presence of an essential singularity at the $\mathrm{X}$-line that is nonevolutionary, ${ }^{21}$ in other words, unphysical. By requiring that such a singularity does not exist, we obtain a formula (Eq. (33)) for the reconnection rate that is similar to that previously obtained by Malyshkin ${ }^{13,14}$ and Kulsrud. ${ }^{15}$ However, unlike their formula, ours does not assume that the diffusion region has a uniform thickness. Our formula also predicts that simply localizing the region of high diffusivity near the X-line is insufficient to ensure a stable, Petschektype solution. The diffusivity profile needs to decrease quadratically with distance away from the X-line. Profiles that are too flat near $x=0$ [e.g., $\left.\eta(x)=\eta_{0} \exp \left(-x^{4} / l_{g}^{4}\right)\right]$ are also unstable.

These results imply a Petschek-type solution can occur only if there is some physical process that creates a diffusion region with a scale length that is smaller than the global scale length of the system. Imposing a spatially localized resistivity is just one of several possible ways a Petschek-type solution can be generated. Using a resistivity model that varies with current density ${ }^{4}$ or an imposed, nonuniform viscosity profile $^{8}$ will also work. Yet another way is to use a nonlocalized resistivity profile in the form of a step function. ${ }^{7}$ There are likely to be even more ways that have yet to be discovered. Whether any of these will be important for real plasmas remains to be seen.

\section{ACKNOWLEDGMENTS}

This work was supported by NSF Grants ATM-0734032 and AGS-0962698, NASA Grants NNX08AG44G and NNX10AC04G to the University of New Hampshire, and subcontract SVT-7702 from the Smithsonian Astrophysical Observatory in support of their NASA Grants NNM07AA02C and NNM07AB07C. D. B. Seaton was supported by PRODEX Grant C90193 managed by the European Space Agency in collaboration with the Belgian Federal Science Policy Office, and by Grant FP7/2007-2013 from the European Commission's Seventh Framework Program under the agreement eHeroes (Project No. 284461). Additional support was provided by the Leverhulme Trust to E. R. Priest. 


\section{APPENDIX A: ITERATION OF EXTERNAL FIELD}

To first order, the parallel field component, $B_{a}^{*}$, just outside the current layer depends on both the transverse field $b$ and the current layer thickness, $a$. For the Green-Syrovatskii model, $B_{a}^{*}$ is

$$
B_{a}^{*}(x)=-\frac{1}{\pi} \sqrt{\frac{1+x}{1-x}} \int_{-1}^{1} \frac{b_{a}(\tau)+\tau}{\tau-x} \sqrt{\frac{1-\tau}{1+\tau}} d \tau,
$$

where $z=x+i y$ and $b_{a}=b+B_{a}(d a / d x) \cdot{ }^{18}$ Equation (A1) reduces to the zeroth order external field $B_{a}=-\sqrt{1-x^{2}}$ when $b_{a}$ is set to zero. Substitution of the numerical solution shown in Figure 3 into (A1) yields the results shown in Figure 10.

The iterated field $B_{a}^{*}$ has a reduced magnitude, because it includes the correction for the conversion of the parallel component, $B_{a}$, into the transverse component, $b_{a}$. If the iterated field is subsequently used in Eqs. (22)-(24), we obtain the iterated transverse field $b_{a}^{*}$ shown by the solid curve at the bottom edge of Figure 10. The difference between $b_{a}^{*}$ and $b_{a}$ is approximately of order $M_{A}^{2}$, so it is a second order correction that can be neglected if $M_{A}$ is sufficiently small.
As Figure 10 shows, iteration alters the shape of $B_{a}$ near $x=0$, even when $M_{A}$ is small. Before iteration, the second derivative of $B_{a}$ at $x=0$ is 1.0 , but after iteration it flips sign to -1.2. Since formula (33) for the reconnection rate depends on the second derivative of $B_{a}$ at $x=0$, the effect of iteration could potentially be significant. To determine whether it is a significant, or not, we write (33) as

$$
\begin{aligned}
M_{A}^{* 4}= & \eta_{0}^{2}\left[-5 B_{a 0} B_{a 0}^{\prime \prime} / 6-B_{a 0}^{2} \eta_{0}^{\prime \prime} /\left(4 \eta_{0}\right)-5 B_{a 0}^{\prime \prime} \delta B_{a 0}^{*} / 6\right. \\
& \left.-5 B_{a 0} \delta B_{a 0}^{* \prime \prime} / 6-\left(\eta_{0}^{\prime \prime} / 2 \eta_{0}\right) B_{a 0} \delta B_{a 0}^{*}\right],
\end{aligned}
$$

where $M_{A}^{*}=M_{A}+\delta M_{A}^{*}, B_{a 0}^{*}=B_{a 0}+\delta B_{a 0}^{*}$, and $B_{a 0}^{* \prime \prime}=B_{a 0}^{\prime \prime}$ $+\delta B_{a 0}^{* \prime \prime}$. The double primes indicate second derivatives evaluated at $x=0$, the asterisks $(*)$ indicate iterated quantities, and the $\delta$ 's indicate the changes due to iteration. For the Green-Syrovatskii model, $B_{a 0}=-1$ and $B_{a 0}^{\prime \prime}=1$. For a Gaussian profile, $\eta_{0}^{\prime \prime}=-2 \eta_{0} / l_{g}^{2}$.

To determine the $\delta B^{*}$ correction terms, we use an analytical approximation for $B_{a}^{*}$ developed by Somov and Titov. ${ }^{18}$ This analytical approximation is shown by the dotted curve in Figure 10. It is obtained from (A1) by approximating $b_{a}$ as a linear ramp of the form $b_{a}=-2 M_{A} x / \alpha$ from $x=0$ to $x=\alpha$, and then setting $b_{a}=-2 M_{A}$ from $x=\alpha$ to $x=1$.

This yields

$$
\begin{aligned}
\delta B_{a}^{*}(x) \approx & \sqrt{1-x^{2}}\left[-\left(2 M_{A} / \alpha\right)+\left(8 M_{A} / \alpha \pi\right) \tan ^{-1} \sqrt{(1+\alpha) /(1-\alpha)}\right] \\
& \left.\left.-\left(2 x M_{A} / \alpha \pi\right) \ln \mid\left(\alpha \sqrt{1-x^{2}}\right)+x \sqrt{1-\alpha^{2}}\right) /\left(\alpha \sqrt{1-x^{2}}\right)-x \sqrt{1-\alpha^{2}}\right) \mid \\
& \left.\left.+\left(2 M_{A} / \pi\right) \ln \mid\left(\sqrt{1-x^{2}}\right)+\sqrt{1-\alpha^{2}}\right) /\left(\sqrt{1-x^{2}}\right)-\sqrt{1-\alpha^{2}}\right) \mid
\end{aligned}
$$

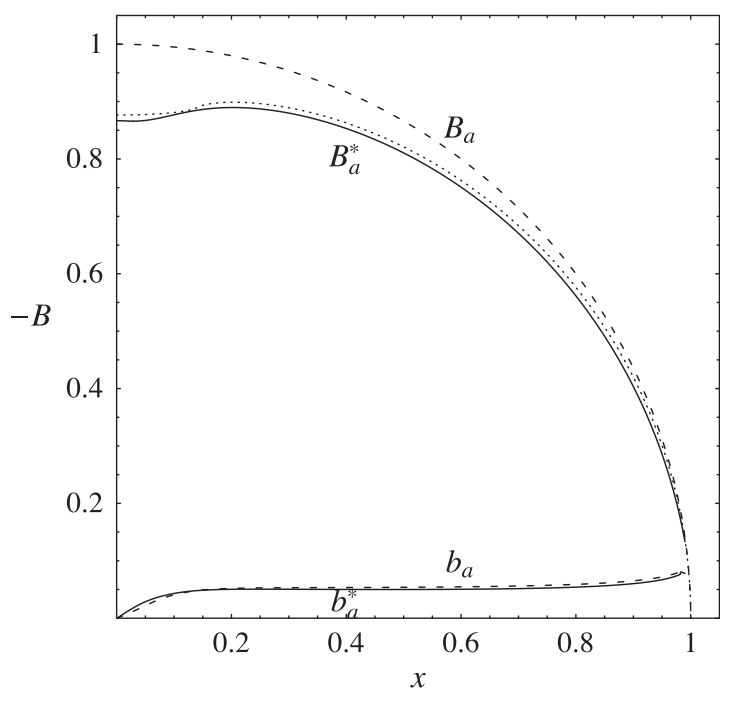

FIG. 10. Comparison of the iterated external fields $B_{a}^{*}$ and $b_{a}^{*}$ (solid curves) with the initial $B_{a}$ and the initial solution for $b_{a}$ (dashed curves). The dotted curve shows Somov-Titov analytical approximation for $B_{a}^{*}$. The values of $\eta_{0}, l_{g}$, and $M_{A}$ are the same as for Figure 3 . where $\alpha=\eta_{0} / M_{A}^{2}$ and $M_{A}$ is prescribed by (33). For small $\alpha$, $\delta B_{a 0}^{*}$ and $\delta B_{a 0}^{* \prime \prime}$ are approximately

$$
\begin{aligned}
& \delta B_{a 0}^{*} \approx\left(4 M_{A} / \pi\right)\left[1-\ln \left(l_{g} / \sqrt{2}\right)\right] \text { and } \\
& \delta B_{a 0}^{* \prime \prime} \approx-2 M_{A} /\left(\pi l_{g}^{2}\right),
\end{aligned}
$$

where $\alpha \approx \sqrt{2} l_{g}$. The $\delta B_{a 0}^{* \prime \prime}$ term in (A2) always remains smaller by a factor of $M_{A}$ than the last term, so the formula for $M_{A}$ is not sensitive to the change in the shape of $B_{a}$ as long as $M_{A} \ll 1$. For the case shown in Figure 10 , with $\eta_{0}=10^{-4}$ and $l_{g}=0.1$, the iterated $M_{A}^{*}$ is 0.0246 . This value is only $7.8 \%$ smaller than the original, uncorrected value of 0.0267 .

By setting $\left|\delta M_{A}^{*}\right| \approx M_{A}$ in (A2), we can determine an upper limit for $M_{A}$ beyond which iteration is needed. For small $l_{g}$, all but the second and last terms of (A2) can be neglected. Upon substitution of the approximate expressions for $\delta B_{a 0}^{*}$ and $\delta B_{a 0}^{* \prime \prime}$, we obtain the limiting condition

$$
M_{A} \leq(\pi / 16) / \mathrm{W}\left(\sqrt{\pi^{2} e / 128 \eta_{0}}\right),
$$

where $\mathrm{W}$ is the product log function (also called the Lambert $\mathrm{W}$ function or the omega function). For $\eta_{0}=10^{-4}$, $M_{A} \leq 0.07$. The upper limit in this case is only 7 times 
greater than the corresponding Sweet-Parker value of $\sqrt{\eta_{0}}=0.01$. For $\eta_{0}=10^{-8}, M_{A} \leq 0.03$. Here the upper limit is 300 times greater than the Sweet-Parker value, but at such a low value of $\eta_{0}$ the flow might become turbulent.

\section{APPENDIX B: PROOF OF ESSENTIAL SINGULARITY AT $X=0$}

In real analysis, the analyticity of a function is defined in terms of the properties of its Taylor expansion. In order to be analytic at a particular point, $x_{0}$, the function must be equal to its Taylor expansion at $x_{0}$ within a finite, nonzero range. If the function is nowhere equal to its Taylor expansion except at $x=x_{0}$, and if its value at $x_{0}$ is finite, then the function has an essential singularity at $x_{0}$. We now prove that for uniform $\eta$ and $B_{a}$, the Taylor expansion of $V(x)$ diverges for any $x>0$ by showing that the coefficients in the power series expansion (28) have the property that

$$
\lim _{n \rightarrow \infty}\left|V_{2 n+3}\right|=\infty .
$$

Since a series cannot converge unless its infinite order term goes to zero, the Taylor expansion will necessarily diverge for any value of $x>0$.

Let $\eta$ be uniform and let $x$ be normalized to the length $\alpha$ instead of $L$. Equations (30) and (31) then simplify to

$$
V_{1}=1, \quad V_{2 n+3}=-2 \sum_{i=0}^{n}(i+1) V_{2 i+1} V_{2(n-i)+1},
$$

where $n$ ranges from 0 to $\infty$. For $n=0$ through 3 ,

$n=0: \quad V_{3}=-2\left(V_{1} V_{1}\right)=-2$,

$n=1: \quad V_{5}=-2\left(V_{1} V_{3}+2 V_{3} V_{1}\right)=12$,

$n=2: \quad V_{7}=-2\left(V_{1} V_{5}+2 V_{3} V_{3}+3 V_{5} V_{1}\right)=-112$,

$n=3: \quad V_{9}=-2\left(V_{1} V_{7}+2 V_{3} V_{5}+3 V_{5} V_{3}+4 V_{7} V_{1}\right)=1360$.

Note that $\left|V_{k}\right| \geq 1$ for all $k$, and that all terms in the equation for $V_{k}$ have the same sign for a given value of $n$ (all negative for $n$ odd, all positive for $n$ even). For any given $n$, the term with the largest absolute value is the last term of the series, and the term with the next to largest value is the first term. Thus, every coefficient $V_{2 n+3}$ must be larger than the coefficient in the power series $G(x)=G_{1} x+G_{3} x^{3}+\cdots$ whose coefficients $G_{2 n+3}$ consist of only the first and last terms of the series that defines the $V_{2 n+3}$ coefficients. In other words, $\left|V_{2 n+3}\right| \geq\left|G_{2 n+3}\right|$ where

$$
\begin{array}{ll}
G_{1}=1, & G_{3}=-2 G_{1} G_{1}, \\
G_{2 n+3}=-2(n+2) G_{2 n+1} G_{1}, & \text { for } n>0 .
\end{array}
$$

If the series for $G(x)$ diverges, so must the series for $V(x)$, since every term of the $V$ series is greater than or equal to the corresponding term of the $G$ series. The recursive formula (B2) for $G_{2 n+3}$ can be simplified to

$$
\left|G_{2 n+3}\right|=(n+2) ! 2^{n} \quad \text { for } n \geq 0 .
$$

Thus,

$$
\lim _{n \rightarrow \infty}\left|G_{2 n+3}\right|=\infty,
$$

which proves (B1). Since $V(0)$ is finite (i.e., 0 ), the singularity at $x=0$ is an essential singularity.

\section{APPENDIX C: DETERMINATION OF CHARACTERISTIC SPEEDS}

For $B_{a}=-1,(19)-(21)$ reduce to

$$
\begin{gathered}
a_{t}=-(a V)_{x}-b V+\eta / a, \\
b_{t}=-(b V)_{x}+(\eta / a)_{x}, \\
(a V)_{t}=-\left(a V^{2}\right)_{x}-b .
\end{gathered}
$$

By rewriting $(a V)_{t}$ as $V a_{t}+a V_{t}$ and using (C1) to eliminate $a_{t}$, we can rewrite $(\mathrm{C} 3)$ as

$$
V_{t}=-V V_{x}+b\left(V^{2}-1\right) / a-V \eta / a^{2} .
$$

The second term on the right-hand side of (C4) goes to zero when $V=1$ because the magnetic field cannot accelerate the plasma beyond the Alfvén speed (i.e., 1 in dimensionless units). We can now rewrite (C1), (C2), and (C4) in matrix form as

$$
\begin{aligned}
& \left(\begin{array}{lll}
1 & 0 & 0 \\
0 & 1 & 0 \\
0 & 0 & 1
\end{array}\right)\left(\begin{array}{l}
a \\
b \\
V
\end{array}\right)_{t}+\left(\begin{array}{ccc}
V & 0 & a \\
\eta / a^{2} & V & b \\
0 & 0 & V
\end{array}\right)\left(\begin{array}{l}
a \\
b \\
V
\end{array}\right)_{x} \\
& =\left(\begin{array}{c}
-b V+\eta / a \\
\eta_{x} / a \\
b\left(V^{2}-1\right) / a-V \eta / a^{2}
\end{array}\right) .
\end{aligned}
$$

The characteristic speeds of the system are given by the eigenvalues $\lambda$, which are the roots of

$$
\left|\begin{array}{ccc}
V-\lambda & 0 & a \\
\eta / a^{2} & V-\lambda & b \\
0 & 0 & V-\lambda
\end{array}\right|=0
$$

or simply $(V-\lambda)^{3}=0$. The three eigenvalues are all real and equal to $V$. Therefore, the system is hyperbolic for all $x$ and $t$. Also, since the eigenvalues are triply degenerate, all information is carried at the advection speed, $V$.

${ }^{1}$ H. E. Petschek, in The Physics of Solar Flares, edited by W. N. Hess (NASA, SP-50, 1964), p. 425.

${ }^{2}$ M. Yan, L. C. Lee, and E. R. Priest, J. Geophys. Res. 97, 8277, doi:10.1029/92JA00170 (1992).

${ }^{3}$ D. A. Uzdensky and R. M. Kulsrud, Phys. Plasmas 7, 4018 (2000).

${ }^{4}$ M. Ugai, Plasma Phys. Controlled Fusion 26, 1549 (1984).

${ }^{5}$ M. Scholer, Geophys. Astrophys. Fluid Dyn. 62, 51 (1991)

${ }^{6}$ N. V. Erkaev, V. S. Semenov, and H. K. Biernat, Nonlinear Processes Geophys. 9, 131 (2002).

${ }^{7}$ H. Baty, T. G. Forbes, and E. R. Priest, Phys. Plasmas 16, 012102 (2009).

${ }^{8}$ H. Baty, E. R. Priest, and T. G. Forbes, Phys. Plasmas 16, 060701 (2009).

${ }^{9}$ B. Roberts and E. R. Priest, J. Plasma Phys. 14, 417 (1975).

${ }^{10}$ T. G. Forbes and E. R. Priest, Rev. Geophys. 25, 1583, doi:10.1029/ RG025i008p01583 (1987). 
${ }^{11}$ E. N. Parker, J. Geophys. Res. 62, 509, doi:10.1029/JZ062i004p00509 (1957).

${ }^{12}$ A. M. Soward and E. R. Priest, Philos. Trans. R. Soc. London, Ser. A 284, 369 (1977).

${ }^{13}$ L. M. Malyshkin, T. Linde, and R. M. Kulsrud, Phys. Plasmas 12, 102902 (2005).

${ }^{14}$ L. M. Malyshkin and R. M. Kulsrud, Phys. Scr. T142, 014034 (2010).

${ }^{15}$ R. M. Kulsrud, Phys. Plasmas 18, 111201 (2011).

${ }^{16}$ V. M. Vasyliunas, Rev. Geophys. 13, 303, doi:10.1029/RG013i001p00303 (1975).

${ }^{17}$ B. V. Somov, V. S. Titov, and A. I. Verneta, Itogi Nauki Tekh. Ser. Astron. 34, 136 (1987) (in Russian).

${ }^{18}$ B. V. Somov, Physical Processes in Solar Flares (Kluwer, Dordrecht, 1992).

${ }^{19}$ V. S. Titov, in Physics of Solar Flares, edited by B. V. Somov (IZMIRAN, Moscow, 1985), p. 141.

${ }^{20}$ V. S. Titov, "Magnetic reconnection in a high-temperature solar plasma," M.S. thesis (Moscow Institute of Physics and Technology, 1985) (in Russian).
${ }^{21}$ B. V. Somov, Plasma Astrophysics Part I: Fundamentals and Practice (Spinger, New York, 2006).

${ }^{22}$ R. M. Green, in Solar and Stellar Magnetic fields, edited by R. Lüst (North-Holland Publishing Co., Amsterdam, 1965), p. 398.

${ }^{23}$ S. I. Syrovatskii, Sov. Phys. JETP 33, 933 (1971) (Engl. Transl.).

${ }^{24}$ D. B. Seaton and T. G. Forbes, Astrophys. J. 701, 348 (2009).

${ }^{25}$ K. Liffman, Publ. - Astron. Soc. Aust. 18, 267 (2001).

${ }^{26}$ R. D. Blandford and M. J. Rees, Mon. Not. R. Astron. Soc. 169, 395 (1974).

${ }^{27}$ S.-H. Kim and A. C. Raga, Astrophys. J. 379, 689 (1991).

${ }^{28}$ H. Baty, E. R. Priest, and T. G. Forbes, Phys. Plasmas 13, 022312 (2006).

${ }^{29}$ E. R. Priest and T. G. Forbes, Magnetic Reconnection: MHD Theory and Applications (Cambridge University Press, Cambridge, 2000).

${ }^{30}$ E. R. Priest and T. G. Forbes, J. Geophys. Res. 91, 5579, doi:10.1029/ JA091iA05p05579 (1986).

${ }^{31}$ N. A. Murphy and C. R. Sovinec, Phys. Plasmas 15, 042313 (2008).

${ }^{32}$ S. Schreier, Compressible Flow (Wiley, New York, 1982). 